\title{
A Generic Method of Line Matching for Central Imaging Systems under Short-Baseline Motion
}

\author{
Saleh Mosaddegh ${ }^{1}$, David Fofi ${ }^{1}$, and Pascal Vasseur ${ }^{2}$ \\ 1 Le2i UMR CNRS 5158, Université de Bourgogne, Le Creusot, France \\ ${ }^{2}$ MIS, Université de Picardie Jules Verne, Amiens, France
}

\begin{abstract}
Line matching across images taken by a central imaging system (perspective or catadioptric) with focus on short baseline motion of the system is proposed. The relationship between images of lines on unitary sphere is studied and a simple algorithm for matching lines are proposed assuming the rotation of the system is known apriori or it can be estimated from some correspondences in two views. Two methods are discussed for retrieving $R$ in the case it is not known apriori. Experimental results on both synthetic and real images are also presented.
\end{abstract}

\section{Introduction}

Line Matching is simply finding the corresponding images of the same 3D line across two or multiple images of a scene. It is often the first step in the reconstruction of scenes such as an urban scene. The images can be captured by any types of imaging systems. This work address central imaging systems e.g. a perspective camera or a catadioptric system. Our method for line matching consists of 3 main steps. First, lines of interest have to be detected in both images. Second, the line segments are projected from 2D to 3D by lifting to unitary sphere. Locating corresponding lines using the relation derived in the following section is the final stage of the line matching algorithm. The geometric relation between two images required a priori here is the rotation of imaging system. This can directly be recorded during image acquisition or later by different available methods such as methods based on matching corresponding vanishing points $[9,10]$. In this work, we are interested in the last step of the matching algorithm. In $[11,15]$ we presented a pipeline for automatic line matching with focus on paracatadioptric systems under the short-baseline motion of the system by employing line intersections correspondences as input to RANSAC in order to compute the rotation of the imaging system. In this work however we employ two different methods for estimating rotation; one is an already developed and robust method of retrieving the rotation using vanishing points direction and second one is a simple alternative method which will shortly be explained. We aim to formulate a generic method of matching lines for all central imaging systems under the short-baseline motion including perspective cameras. The latest work which significantly improves on the state of the art for line matching across two or more perspective views can be found in [1]. They also assume that the

P. Foggia, C. Sansone, and M. Vento (Eds.): ICIAP 2009, LNCS 5716, pp. 939-948 2009.

(C) Springer-Verlag Berlin Heidelberg 2009 
motion of the camera is apriori known (through fundamental matrix) and the problem is then reduced to finding corresponding lines by help of fundamental matrix and epipolar geometry. The most related work to line matching in catadioptric imaging systems is done in [5] for visual servoing/tracking purposes by tracking line features during the camera (and/or the object) motion. The paper is mainly concerned with the use of projected lines extracted from central catadioptric images as input of a visual servoing control loop. The method is based on the estimation of the partial camera displacement between two views, given by the current and desired images and it is entirely different from the problem of matching lines across views in which there is considerable motion between corresponding lines which is the subject of our work. While in the perspective case line matching is rather efficiently solved, to the best of our knowledge, this is the first work dealing with this problem in catadioptric images.

\section{Proposed Method}

The rest of this text is organized as follows. In the next section, we derive the relation between normal vector of the great circle of any 3D line represented in the first unitary sphere coordinate system and its corresponding vector expressed in the second system which in return gives us an adequate tool to match lines. Then, we recall the sphere equivalence followed by a brief description of the two algorithms for recovering the rotation of the imaging system. We skip giving the definition of catadioptric imaging systems and unitary sphere model due to space restrictions. General readers are referred to references $[2,3,4]$ for explanations and details. Finally some experimental results of applying the proposed algorithm on both synthetic and real images are presented. For extracting line segments, we use the line extraction algorithm of [6]. For recovering $R$, along with our proposed method we also explain attitude estimation method of [10]. We will shortly give a brief explanation of these algorithms.

\subsection{The Relation between Images of 3D Lines on Unitary Sphere}

Having the intrinsic parameters of the imaging system, the key idea is to project the image on the unitary sphere, turning the conic curves (images of the lines on the image plane) into their corresponding great circles on the unitary sphere. Knowing that a great circle is fully defined by normal vector of its plane, the problem of matching conics is then reduced to matching these vectors. In this section we show that under short range motion, two corresponding great circle are mainly related by rotation part of the imaging system motion. Consider a line in $3 \mathrm{D}$ scene with two separate $3 \mathrm{D}$ points $X_{1}$ and $X_{2}$ on it. Suppose $n_{1}$ is the non-normalized vector of the plane which passes through these two points and the origin of the first unitary sphere and $n_{2}$ is corresponding vector expressed in the second model (Fig. 1). Then:

$$
\begin{gathered}
n_{2}=\left(R X_{1}+t\right) \times\left(R X_{2}+t\right)=\operatorname{det}(R) R^{-t}\left(X_{1} \times X_{2}\right)+[t]_{\times} R\left(X_{1}-X_{2}\right)= \\
\operatorname{det}(R) R^{-t} n_{1}+[t]_{\times} R\left(X_{1}-X_{2}\right)=R n_{1}+[t]_{\times} R\left(X_{1}-X_{2}\right)
\end{gathered}
$$


Where metric transformation of the imaging system (represented by two unitary spheres in Fig. 1) is defined by the rotation matrix $R$ and translation vector $t$ and $R^{-t}$ is inverse transpose of $R$. Note that for a rotation matrix, $\operatorname{det}(R)=1$ and transpose inverse is the same as $R$. Above relation coincides with the relation obtained in $[7,8]$ in which equivalent Euclidean Plücker representation of the line is used to derive a similar formula:

$$
n_{2}=R n_{1}+[t]_{\times} R l, l=\frac{\left(X_{1}-X_{2}\right)}{\left\|X_{1}-X_{2}\right\|}
$$

Where the 3D line segment is represented by its infinite supporting line represented by two vectors $l$ and $n$. $l$ is a unit vector parallel to the line, and $n$ is a non-normalized vector to the plane defined by the line and the origin of the coordinate system and its norm is equal to the distance of the line to the origin, e.g. $\|n\|=d$, see Fig. 1. Therefore if transformation between two positions of the imaging system is a pure rotation $(t=0)$ or the movement of the system in comparison to its distance to the scene is very small (short baseline, for example aerial imaging), we can neglect the second term in the above equations and conclude that under the pure rotation or short base line motion, $n_{1}$ and $n_{2}$ are related by the rotation matrix:

$$
n_{2}=R n_{1}
$$

This equation can also be visually verified as shown in Fig 2. One immediate result is that for the case of short-baseline, after estimating the rotation matrix, for each line in the first image, all which is needed to find its corresponding line in the second image is to multiply $R$ at normal vector of great circle of the line. The result vector is pointing at the same direction as the normal vector of great circle of corresponding line is pointing (inside a reasonable angular distance error).

\subsection{Lifting from Image Plane to Unitary Sphere}

The necessary equations for lifting a pixel on the image onto the unitary sphere are already derived in several slightly different formulations in literature. We use

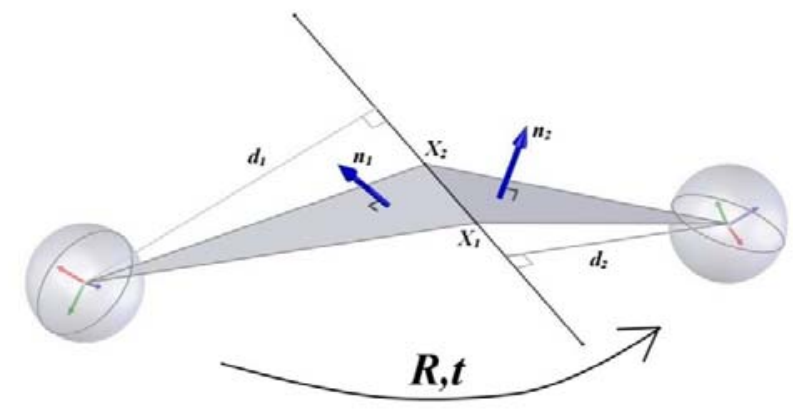

Fig. 1. A 3D Line in the scene and its projections on a unitary sphere at two different positions. $n_{1}$ and $n_{2}$ are normal vectors of related great circles. 


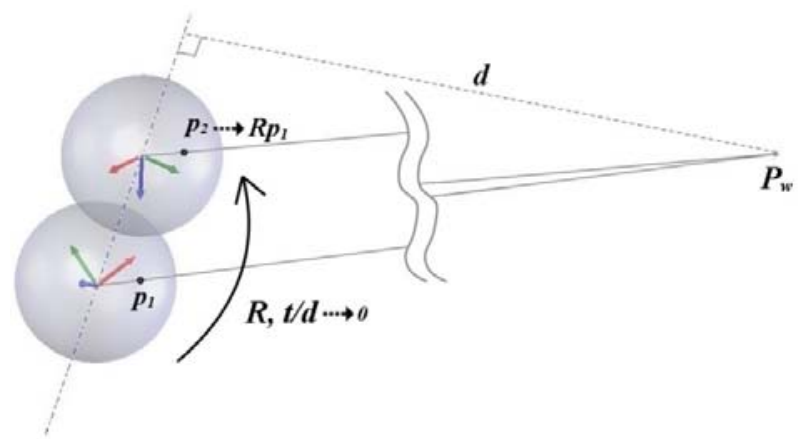

Fig. 2. If the motion of the imaging system is a short range motion (pure rotation), the images of a world point on the unitary sphere at two different positions are approximately (absolutely) related by the rotation of the system. Note that there is a considerable arbitrary rotation between two unitary sphere coordinate systems.

the projection model of [14] which is an extension of the model proposed by [12, 13] as follows:

Consider the pixel $(i, j)$ on the image plane( of our interest are the pixels belonging to conic segments, the images of $3 \mathrm{D}$ lines on the image plane). The pixel coordinates after lifting are:

$X_{s}=\Omega \times m, \quad Y_{s}=\Omega \times n, \quad Z_{s}=\Omega-\xi$

where $\Omega=\frac{\xi+\sqrt{1+\left(1-\xi^{2}\right)\left(i^{2}+j^{2}\right)}}{i^{2}+j^{2}+1}, \quad n=\frac{i-v_{0}}{\gamma r}, \quad m=\frac{j-u_{0}-n s \gamma}{\gamma}$

$\xi$ is a parameters describing the mirror shape, $s$ is skew parameter, $r$ is aspect ratio, $\gamma$ is generalized focal lengths and $\left(u_{0}, v_{0}\right)$ is principal point . All these parameters are available as the result of calibration. The same above equations can be used in reverse to project a point on unitary sphere into image plane.

\subsection{Recovering $R$}

There are several methods for estimating $\mathrm{R}$ applicable to all types of central imaging systems (cf. [9] for a review on these methods and their pros and cons). Regarding the simplicity and robustness we have experimented with two automatic methods, one from [9] which works in urban scene with at least two groups of $3 \mathrm{D}$ parallel lines and the other one is our proposed method which is suitable for short-baseline motion as follows.

Recovering $\boldsymbol{R}$ using Vanishing Points. Vanishing Points (VP) are points on the plane at infinity and therefore they are invariant to translation. A rotation matrix has three degree of freedom and each vanishing point correspondence provides two rotation constraints. Therefore for estimating $R$, it is sufficient to have two enough distinct VP correspondences in the two views. Reference [10] has exploited above facts to estimate the rotation of an imaging system in 

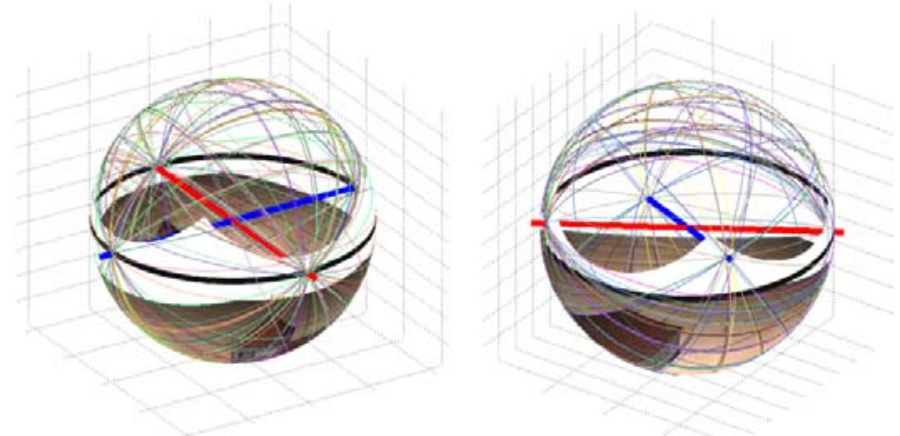

Fig. 3. Projection of two paracatadioptric images on the unitary sphere, their extracted great circles and two dominant vanishing directions. For a better demonstration, half of the great circles are hidden.

two steps, extraction of vanishing points followed by recovering $R$ by matching these points. For the sake of completeness, we briefly explain their method for extracting VP. For matching part, interested readers are referred to [9]. Consider two great circles corresponding to two parallel 3D lines. The intersection of these two great circles (say vector $u$ ) corresponds to the common direction of the lines. $u$ should also point at the direction of any other line parallel to these two lines (inside a similarity threshold). Therefore checking for all lines, we can find the number of lines that may share the same direction $u$. Repeating this procedure for each combination of two great circles we can compute the vector that corresponds to the highest number of parallel lines (the vanishing point of those lines). To detect the second dominant direction, we remove the lines belong to the first dominant direction and repeat the above steps (See Fig 3).

Recovering $\boldsymbol{R}$ using points correspondences and RANSAC. The idea behind this approach is already depicted in Fig 2. In the case of short-baseline motion, the image of the scene on the unitary sphere goes under the same rotation as imaging system. Exploiting this fact, we suggest the following simple method for extracting the $R$ :

- Manually or by means of automatic algorithms such as SIFT feature extractor, extract enough point correspondences between two views.

- Lift these correspondences to their unitary spheres.

- Using RANSAC or similar fitting algorithms, find the best rotation matrix which relates these corresponding points.

Note that this method is only feasible when the imaging system goes under a short range motion. Note also that theoretically, having the images of two salient 3D points (which are not collinear with the center of unitary sphere) and their correspondences is sufficient to estimate the rotation matrix. However we employ RANSAC to be able to automatically extract some interest points and their correspondences without being concerned about the errors in detection of positions of these points in the image planes and also any possible mismatches. 


\section{Implementation Details and Experimental Results}

The proposed method is composed of the following main steps:

1. Given two images taken by a central imaging system under short range motion, extract their line segments (cf. [6]).

2. Extract two dominant vanishing directions and use them to estimate $R$ (cf. section 2 and [9]).

3. Project line segments onto the unitary sphere and compute normal vectors of their great circles.

4. Find corresponding great circle of each line segment in the other image by using the relation (1) derived in section 2.1.

Fig. 4 along with Fig. 3 demonstrates the steps of our algorithm on a pair of synthetic images. We have applied the $R$ on whole first sphere for the sake of demonstration. In practice and during the implementation only normal vector and two end points of each segment (necessary to find the segment bounding box for the case there are ambiguities) are affected. In the last step, for some segments, matching great circles using the relation (1) is not enough and one ambiguity may occur when more than one segment are lying on corresponding infinite line because these segments are all located on the same 3D scene plane (as it is shown in Fig. 5). To resolve this ambiguity we also find the corresponding bounding box of the segment in the first image and we chose the candidate segment which is inside the bounding box or is intersecting it. During our experiments, the calibration method of [14] was employed to calibrate the imaging system. As the first approach we used the framework of [2] to detect the lines, extract the vanishing points and estimate $R$. This approach is especially of interest since the method uses already extracted lines (great circles on the unitary sphere) to estimate $R$ in comparing to the second approach which includes extra steps of extracting salient point correspondences and fitting a rotation matrix to them. Therefore in our experiments, we used the first approach since we had enough vanishing points available.

Synthetic images. Fig. 5 shows the line segments extracted on two synthetic aerial images (upper row) and matched lines (lower row). There is a 110 degree rotation around the optical axis of the system and 30 and 20 degree around two other axes all measured w.r.t. a fixed coordinate system. Translation of the system is neglectable. Only lines of length 15 pixels or more are considered. The angular threshold for matching great circles is set to one degree. 261 and 226 segments are obtained for the left and right images, respectively. The algorithm outputs the matches displayed at the bottom row of the figure. All of the 121 matches obtained are correct.

Real Images. Fig. 6 shows the result of applying our algorithm on two real perspective images. Note that this method is far simpler than an approach such in [1] in which photometric properties of the segments neighborhoods along with 


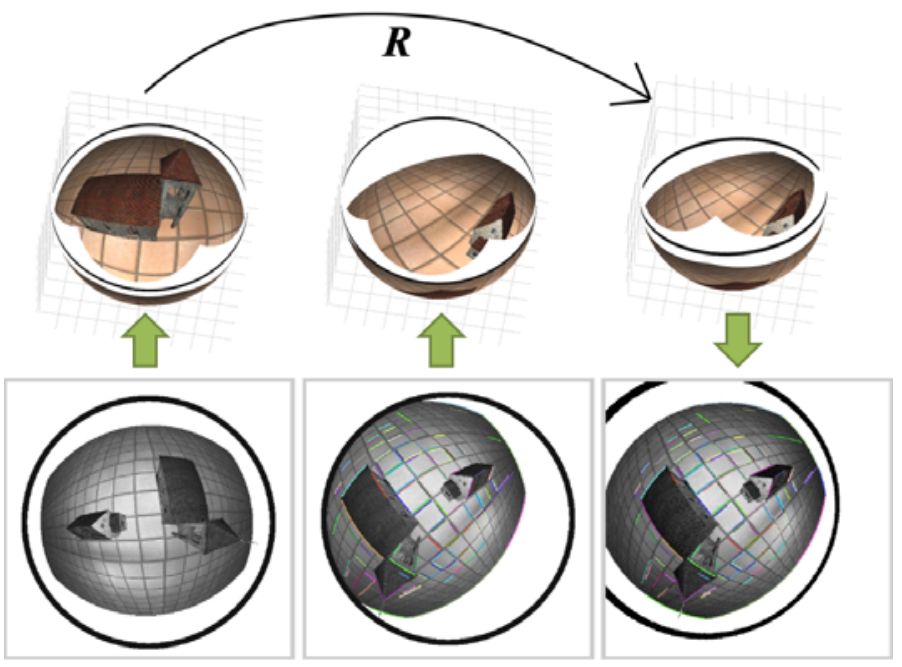

Fig. 4. Steps of the proposed algorithm: Lifting images onto unitary sphere, recovering $R$ and rotating the first image according to $R$. Segments on the back projected image now coincident with their correspondings in the second image.
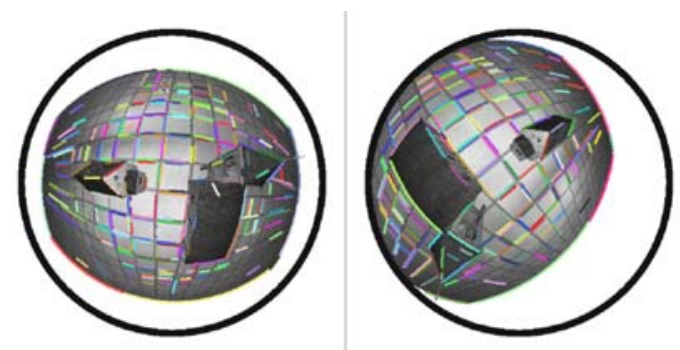

(a)
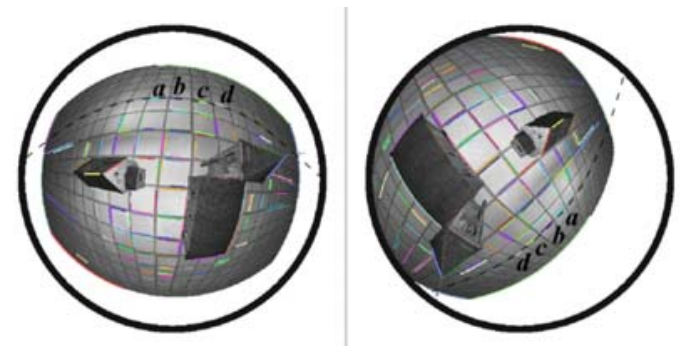

(b)

Fig. 5. Top: Two paracatadioptric images and their extracted segments. Bottom: matched lines (each color represents one correspondence. All of the 121 matches shown are correct. Note that segments $a, b, c$ and $d$ share the same great circle (dashed line). The end points of each segment are used to find the correct correspondence. 

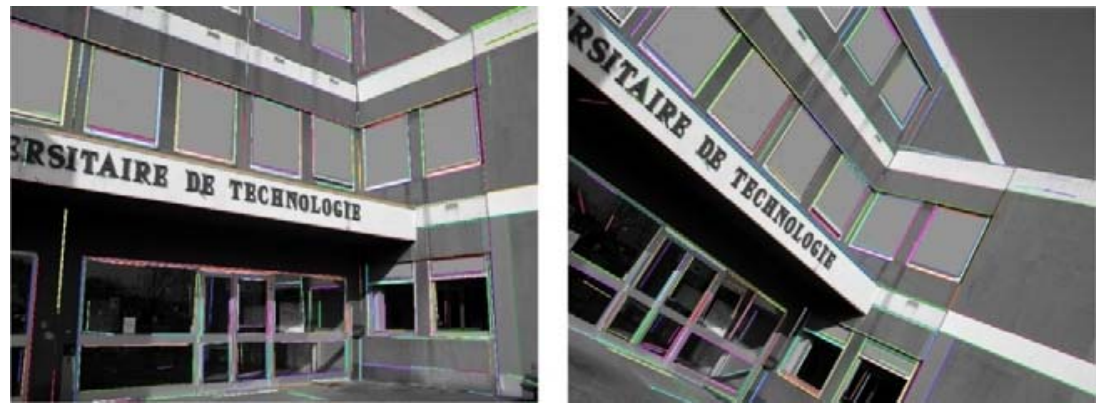

(a)
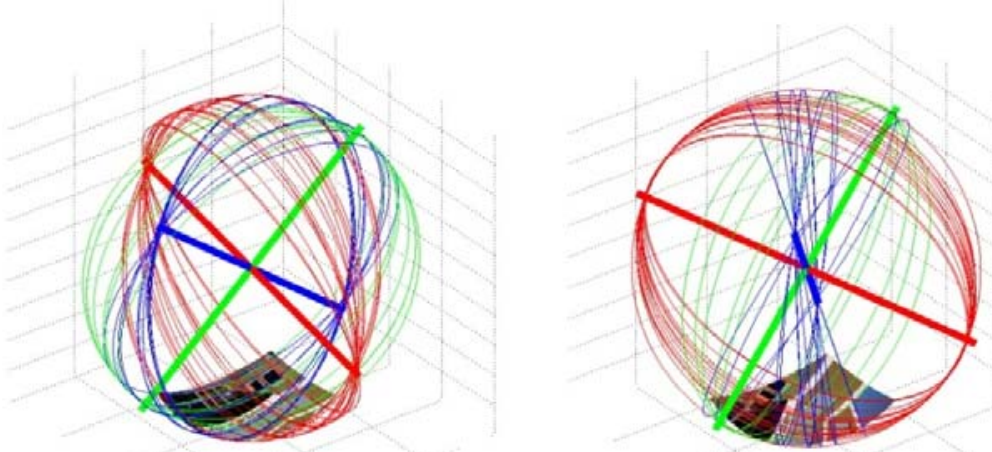

(b)
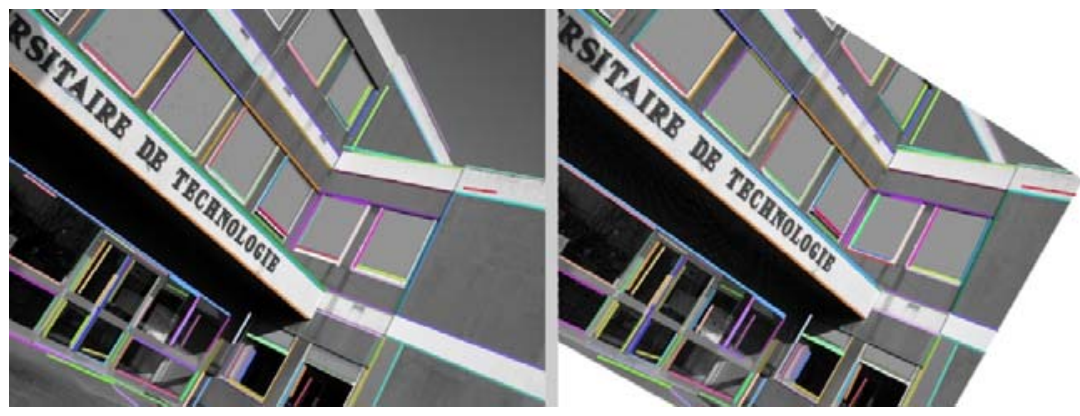

(c)

Fig. 6. Top: Two perspective images and their extracted segments. Middle: related great circles onto unitary sphere and three dominant vanishing directions $(75 \%$ of lines are hidden). Bottom: matched lines (each color represents one correspondence. Note that the image on the left is the second view and the image on the right is the first view after applying the $R$ on it). $93 \%$ of 129 matches shown are correct. 
epipolar geometry are exploited to do the same job. For this experiment, rotating imaging system around its focal point was not easy since this point is somewhere inside the camera and we needed a special flexible fixture to carry out the job. However, rotating (while trying to avoid translating) of imaging system can be considered as a short-baseline motion. Images of Fig. 6 are taken by a random rotation of the imaging system in this way. Even though matching 2 vanishing points is enough to recover $R$, we use 3 vanishing points to reduce the overall error. The recovered $R$ is composed of an approximately 31 degree rotation around the optical axis of the system and 4 degree and 4.5 degree around two other axes all measured w.r.t. a fixed coordinate system. For this example, the numbers of segments extracted are 284 and 245 for the left and right images. Obtained Matches are shown at the bottom row of the figure. 93\% of the 129 matches are correct. The performance of the proposed method has decreased not only because the motion of the system is not a real short-baseline motion (we found that there is also a small translation of the imaging system) but mainly because perspective imaging systems suffer from a limited field of view. The wider field of view of a perspective camera results in the better extraction of lines and the longer line segments. Note that the larger error in the computing the position of the lines causes larger error in the estimation of the vanishing points and therefore a less accurate recovery of $R$ and eventually more mismatches. The second method of recovering $R$ by matching salient points rather than vanishing points, however, can overcome this problem since it does not depend on the images of the lines.

\section{Conclusion}

This work dealt with the problem of matching lines for all types of central imaging system under a short baseline motion by presenting a generic and simple line matching approach. The method is composed of three main steps of extracting line segments, recovering $R$ and matching lines respectively. Two methods for retrieving $R$, one based on matching vanishing points and the other based on matching any two feature points were also presented. The evaluation and optimization of the method on the real catadioptric systems and extension of the method to long baseline motion are the subjects of future works.

\section{References}

1. Schmid, C., Zisserman, A.: Automatic line matching across views. In: Proceedings of the IEEE Conference on Computer Vision and Pattern Recognition, pp. 666-672 (1997)

2. Barreto, J.P., Araujo, H.: Issues on the geometry of central catadioptric image formation. In: International Conference Computer Vision 01, Hawaii, pp. 422-427 (2001)

3. Baker, S., Nayar, S.: A theory of catadioptric image formation. In: Proc. of IEEE International Conference on Computer Vision, Bombay, pp. 35-42 (1998) 
4. Geyer, C., Daniilidis, K.: Catadioptric projective geometry. International Journal of Computer Vision 45(3), 223-243 (2001)

5. Mezouar, Y., Haj Abdelkader, H., Martinet, P., Chaumette, F.: Central catadioptric visual servoing from $3 \mathrm{~d}$ straight lines. In: IEEE/RSJ Int. Conf. on Intelligent Robots and Systems, IROS 2004, Sendai, Japan, September 2004, vol. 1, pp. 343-349 (2004)

6. Bazin, J.C., Demonceaux, C., Vasseur, P.: Fast central catadioptric line extraction. In: Martí, J., Benedí, J.M., Mendonça, A.M., Serrat, J. (eds.) IbPRIA 2007. LNCS, vol. 4478, pp. 25-32. Springer, Heidelberg (2007)

7. Navab, N., Faugeras, O.D.: The Critical Sets of Lines for Camera Displacement Estimation: A Mixed Euclidean-Projective and Constructive Approach. International Journal of Computer Vision 23(1), 17-44 (1997)

8. Bartoli, A., Sturm, P.: The 3D line motion matrix and alignement of line reconstructions. International Journal of Computer Vision 57(3) (May/June 2004)

9. Bazin, J.C., Kweon, I.S., Demonceaux, C., Vasseur, P.: Spherical region-based Matching of Vanishing Points in Catadioptric Images. In: OMNIVIS 2008, in conjunction with ECCV 2008, Marseille, France. Octobre (2008)

10. Bazin, J.C., Kweon, I.S., Demonceaux, C., Vasseur, P.: UAV Attitude Estimation by Vanishing Points in Catadioptric Image. In: IEEE International Conference on Robotics and Automation 2008 (ICRA 2008), Pasadena, CA, Mai (2008)

11. Mosaddegh, S., Fofi, D., Vasseur, P., Ainouz, S.: Line Matching across Catadioptric Images. In: OMNIVIS 2008, in conjunction with ECCV 2008, Marseille, France (October 2008)

12. Barreto, J.P., Araujo, H.: Issues on the geometry of central catadioptric image formation. In: CVPR, vol. 2, pp. 422-427 (2001)

13. Geyer, C., Daniilidis, K.: A unifying theory for central panoramic systems and practical implications. In: Vernon, D. (ed.) ECCV 2000. LNCS, vol. 1843, pp. 445-461. Springer, Heidelberg (2000)

14. Mei, C., Rives, P.: Single view point omnidirectional camera calibration from planar grids. In: ICRA 2007 (April 2007)

15. Mosaddegh, S.: Line Matching across Catadioptric Images. Master's thesis, Le2i laboratory, Université de Bourgogne, France (June 2008) 